\title{
Analysis of the Influence Factors on the Efficiency of Marine Tail Shaft Transmission System
}

Jian WANG, Zheng Lin LIU, Li ZOU

Wuhan University of Technology, Wuhan 430063, China

\begin{abstract}
The transmission efficiency of the marine tail shaft was calculated with the finite element method and experimentvalidatedthe rationality and feasibility of finite element models. The research conclusions as follows: power consumption increases with the shaft length, shaft diameter and speed increase. The results can provide a theoretical support for further research andit is also helpful for engineering applications.
\end{abstract}

\section{Introduction}

When the ship is sailing on the ocean, itsshaft between the stern bearing and propeller is working in water, which is normally called the shaft in water [1]. There has always been scholars to question the power consumption of the shaft in water and then affect the ship's economy and efficiency. In particular, the shaft in water power consumption of the warship will further affect its operationalperformanceattract more attention from scholars and experts [2,3]. However, it is limited to directly measurethe shaft in water's power consumption in real environmental conditions when theshaft in water runs in the water [4]. So far there has been no relevant studies or reports about the shaft in water drive efficiency. In this paper, firstly, finite element model of the shaft in water is built in GAMBIT and its friction torque in water is calculatedvia FLUENT, and then the power consumed by the resistance is obtained. Our project team designed the relevant design experiments to test the friction torque produced by the shafting in water and then calculate the power consumption. The theoretical results and experimental results are comparatively analyzed to find out the factors affecting the efficiency of the shaft transmission in water.

\section{Friction torque calculation with FEM}

The power consumption caused by friction can be calculated by torque, so the friction torque should be calculated by FLUENT firstly.The power consumptioncan be calculated with:

$$
P=\frac{T \cdot S}{9.55}
$$

Where $P(\mathrm{~W})$ is the power consumption, $S$ is the rotation speed of the shaft and $T$ is the friction torque. The meaning is the same as in the following.

\subsection{Finite elementmodel}

Finite element model of shafting in water was established by the pretreatment software GAMBIT of FLUENT.First of all, establishing open water film axial segment model, and then placing the model used in the experiments of flow field area of the tank. This fluid region is relatively static.A rotating region on the outer cylindrical surface of the shaft is established, which is coaxial with the cylindrical surface. It is assumed that the 
flow field rotates around the $\mathrm{Z}$ axis, which is in a static state with the axial section. The calculation area adopts structured grid, and the water film model is divided into volume grid directly. The finite element model has three kinds of $240 \mathrm{~mm}, 310 \mathrm{~mm}$ and $390 \mathrm{~mm}$ in the diameter of the shaft section. Each diameter of the shaft section corresponds to two lengths of $1200 \mathrm{~mm}$ and $1500 \mathrm{~mm}$. For example, when the axial section of the shaft in water is $1200 \mathrm{~mm}$ and the diameter of the shaft is $240 \mathrm{~mm}$, the diameter of the rotating area is selected $360 \mathrm{~mm}$. Due to the large external flow field, the impact on the friction power consumption is small, the grid partition can be sparse, a total of 44350 units, the rotation of the basin and the axis of contact, the grid division of the higher density, a total of 43350 units.As shown in Figure 1. Thequality inspection of grid division meets the requirements and the finite element models of different axial lengths under different axial lengths are similar to that of example.

In the calculation of the friction powerconsumption of the shaft in the open water, the internal calculation area is only relative axial rotation, there is no mutual interference between other objects and axial segments. The boundary conditions of the inlet is velocity inlet, and outlet boundary pressure outlet and the external surface of the cylinder is set to the wall and the surface between the inner rotating basin and the outer cylinder is set to INTERFACE. When the finite element model is introduced into FLUENT, these two overlapping surfaces are connected together to form a pair of interface, which can realize the data exchange between the external static flow field and the inner rotating basin. At last, the axial section surface is set to the wall.

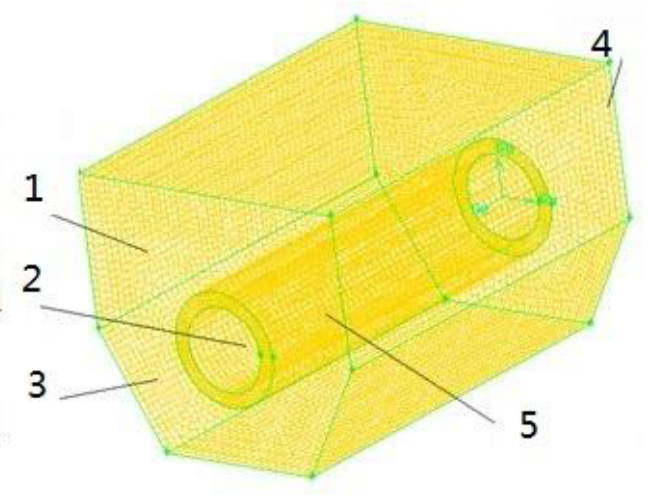

1-External flow field 2-Shaft 3-Inlet4-Outlet

5-Rotational flow field

Figure 1.Finite elementmodel

\subsection{Determine the diameter of rotating basin}

The external flow field can be limited to a certain range when calculating the rotational power consumption in the water, but the prerequisite is to determine the optimum diameter of the shaft section in the model. The external flow field can be limited to a certain range when the length and diameter of the shaft is rotated in the water. The friction torque values of different rotating basins can be obtained by the trial calculation, as shown in Table 1.

Table 1.Torquevalues under different rotating basin diameter

\begin{tabular}{|c|c|c|c|c|c|c|c|}
\hline \multirow{3}{*}{$\begin{array}{l}\mathrm{L} \\
(\mathrm{mm})\end{array}$} & \multirow{3}{*}{$\begin{array}{l}\mathrm{D} \\
(\mathrm{mm})\end{array}$} & \multirow{3}{*}{$\begin{array}{l}\mathrm{D}_{\mathrm{R}} \\
(\mathrm{mm})\end{array}$} & \multicolumn{5}{|c|}{$\mathrm{S}(\mathrm{r} / \mathrm{min})$} \\
\hline & & & 50 & 80 & 110 & 150 & 200 \\
\hline & & & \multicolumn{5}{|c|}{$\mathrm{T}(\mathrm{N} \cdot \mathrm{m})$} \\
\hline \multirow{3}{*}{1200} & \multirow{3}{*}{240} & 360 & 0.4 & 1 & 1.6 & 2.7 & 4.5 \\
\hline & & 450 & 0.4 & 0.9 & 1.6 & 2.7 & 4.4 \\
\hline & & 600 & 0.4 & 1 & 1.6 & 2.8 & 4.5 \\
\hline
\end{tabular}

Where, $L$ is the length of the shaft in water, $D$ is the diameter, $D_{R}$ is the diameter ofrotating basin, $S$ is the rotation speed of the shaft and $T$ is the friction torque.

Obviously, there is no effect on the friction torque calculation of the rotating basin under different coaxial section diameter. According to the calculation formula of the power consumption and the friction torque, the power consumption is not related to the size of the rotating basin, so any diameter can be used in the FEM calculation

\subsection{Calculation results}

The length of the shaft is 1500 and $1200 \mathrm{~mm}$ respectively. The theoretical friction torque and power consumption of the shaft in water at different rotational speeds (50r/min, 80r/min, 110r/min, 150r/min, 200r/min) are shown in Table 2. 


\section{Friction torque measurement}

\subsection{Experimental device}

According to the finite element model, we developed a ratio of 1:1 shaft in watering simulation experimental device which was installed on the comprehensive experimental platform for ship shafting.The friction torque of the shaft is measured in water and air respectively, and then the power consumption was calculated.The output end of the drive motor of the ship shafting integrated experiment platform is equipped with a torque meter to measure the output torque and power of the motor.Measuring the friction torque of the shaft in water, and then measuring the friction torque of the shaft exposed to air. Two experiments should be ensured that running, sealing, lubrication and other conditions unchanged. By comparing the results of two experiments, the power consumption to overcome the resistance of the shaft is obtained.

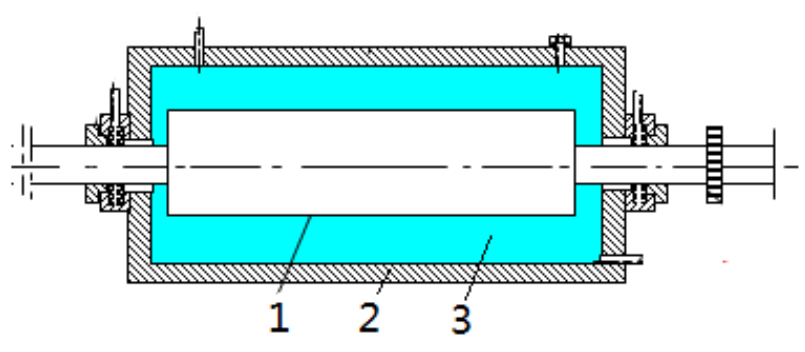

1-Shaft 2-Water tank 3-Water

Figure 2.Experimental device

\subsection{Experimental Results}

Power consumption under different conditions is shown in Table 3, which is the interpolationof the tail shaft power consumption in the water and in the air, that is, power consumed by the shaft to overcome the resistance of water.According to the experimental results, it is concluded that when the length and diameter of the shaft are constant, the power consumption of the shaft increases with the increase of the rotating speed.

Table2. Calculation results with FEM

\begin{tabular}{|c|c|c|c|c|c|c|}
\hline \multirow{3}{*}{$\mathrm{L}(\mathrm{mm})$} & \multirow{6}{*}{$\mathrm{D}(\mathrm{mm})$} & 50 & 80 & 110 & 150 & 200 \\
\cline { 3 - 7 } & & \multicolumn{5}{|c|}{$\mathrm{P}(\mathrm{W})$} \\
\hline \multirow{3}{*}{1200} & 240 & 2.1 & 8.4 & 18 & 42.4 & 94 \\
\cline { 3 - 8 } & 310 & 5.2 & 19.3 & 46 & 107 & 230 \\
\cline { 2 - 7 } & 390 & 9.9 & 36.9 & 91 & 220 & 502 \\
\hline \multirow{3}{*}{1500} & 240 & 2.6 & 8.4 & 20 & 50.3 & 111 \\
\cline { 2 - 7 } & 310 & 6.3 & 22.6 & 57 & 129 & 272 \\
\cline { 2 - 7 } & 390 & 13 & 41.9 & 115 & 267 & 607 \\
\hline
\end{tabular}

Table3. Experimental results

\begin{tabular}{|c|c|c|c|c|c|c|}
\hline \multirow{3}{*}{$\mathrm{L}(\mathrm{mm})$} & \multirow{2}{*}{$\mathrm{D}(\mathrm{mm})$} & \multicolumn{5}{|c|}{$\mathrm{S}(\mathrm{r} / \mathrm{min})$} \\
\cline { 3 - 7 } & & 50 & 80 & 110 & 150 & 200 \\
\cline { 3 - 7 } & & \multicolumn{5}{|c|}{$\mathrm{P}(\mathrm{W})$} \\
\hline \multirow{3}{*}{1200} & 240 & 2.6 & 3.1 & 14.8 & 43.6 & 134.9 \\
\cline { 2 - 7 } & 310 & 9.5 & 36 & 55 & 91.2 & 230 \\
\cline { 2 - 7 } & 390 & 15.4 & 40 & 78 & 221 & 299.6 \\
\hline \multirow{3}{*}{1500} & 240 & 5.5 & 30.4 & 70.6 & 229.4 & 281.4 \\
\cline { 2 - 7 } & 310 & 6.4 & 141.9 & 77 & 231.3 & 308.3 \\
\cline { 2 - 7 } & 390 & 20 & 46.2 & 85.3 & 228.4 & 390.7 \\
\hline
\end{tabular}




\section{Result and discussion}

According to table 2, power consumption curvewith the change of the shaft diameter at the speed of $50 \mathrm{r} / \mathrm{min}$ as shown in figure 3 . With the shaft length increases, shaft in water power consumptionincreases; under the same shaft length and the same speed, with the increase of shaft diameter, power consumption increases too. According to table 2 and table 3 , the theoretical calculation results and experimental results, the relative error remained at about $20 \%$ or less, in accordance with the requirements of engineering applications.

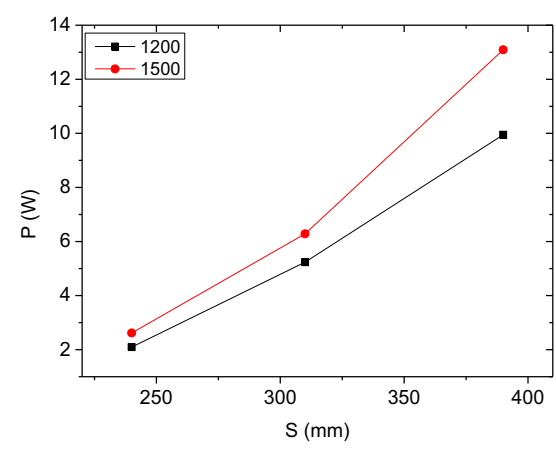

Figure 3.Power consumption varies with diameter

When the shaft length is $1500 \mathrm{~mm}$ and the diameter is $390 \mathrm{~mm}$, the FEM calculation result is compared with the experimental results, as shown in Figure 4.Other comparison results are similar under different operating conditions, and here are no longer cited.

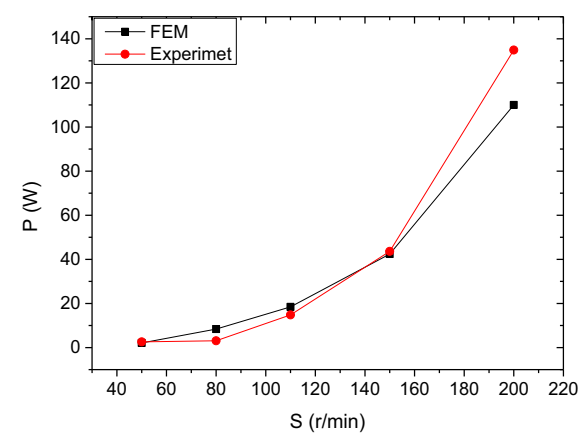

Figure 4. Comparison between experimentationand calculation

Easy to see the various experimental conditions calculated with the resultant power variation and trends basically consistent with the lower section of coaxial rotational speed increases, the corresponding increase in the power shaft section of the water by the rate will slow and fast.

\section{Conclusion}

The following conclusions can be drawn from this research: the experimental results verify the feasibility of the finite element model, and computational results by FLUENT also demonstrate the rationality of experiment. In finite element calculation, the author found that rotating area diameter size had no effect on the friction torque and power consumption, which can provide a theoretical reference for the other simulation. The power consumption of the shaft in water is increased with the increase of the length, diameter and rotational speed of the shaft.

Because of the gap between the experimental model and the actual ship, the experimental model is relatively small, the measured torque and power consumption are relatively small under different conditions. In the follow-up study the author will establish a larger experimental platform to get more experimental data to further explore the impact factor of the shaft in water drive efficiency and power consumption and describe them in a more specific numerical relationship.

\section{Acknowledgment}

This work was financially supported by the project of Natural Science Foundation of China (No. 51379168), Key Natural Science Foundation of China (No. 51139005).

\section{References}

1. Liang X X, Liu Z L, Liu J., Applied Mechanics \& Materials, "Analysis of Pre-Tightening Strength of Ship Tail Shaft Coupling Based on Workbench Software", 496-500:1028-1031(2014).

2. Yao Z S, Xue W P, Song H Q, et al. Journal of Guangxi University, "An experimental study on shift lining structure of frozen shaft in water-rich soft rock", (2014)

3. Zhang G C., Ship Engineering"Application of Energy-Feedback and Energy-saving Technology Based on SINAMICS in the Marine Shaft Generating", (2010)

4. Quan-Chao L I, Zhi-Heng L I., Mechanical Engineer "Research on Parametric Design for Marine Shaft", (2013) 